Revue d'histoire de l'enfance « irrégulière »

Le Temps de l'histoire

Hors-série | 2007

Pages d'histoire, la protection judiciaire des mineurs, $\mathrm{XIX} \mathrm{X}^{\mathrm{e}} \mathrm{XX} \mathrm{X}^{\mathrm{e}}$ siècles

\title{
L'ISES des Sables d'Olonne. Une création de 1954 pour les mineurs difficiles
}

Jacques Bourquin

\section{(2) OpenEdition \\ Journals}

Édition électronique

URL : http://journals.openedition.org/rhei/3119

DOI : 10.4000/rhei.3119

ISBN : 978-2-7535-1647-2

ISSN : $1777-540 \mathrm{X}$

Éditeur

Presses universitaires de Rennes

Édition imprimée

Date de publication : 1 juin 2007

Pagination : 295-305

ISSN : $1287-2431$

Référence électronique

Jacques Bourquin, "L'ISES des Sables d'Olonne. Une création de 1954 pour les mineurs difficiles », Revue d'histoire de l'enfance « irrégulière » [En ligne], Hors-série | 2007, mis en ligne le 01 février 2010, consulté le 02 mai 2019. URL : http://journals.openedition.org/rhei/3119; DOI : 10.4000/rhei.3119 


\section{L'ISES des Sables d'Olonne. Une création de 1954 pour les mineurs difficiles ${ }^{(*)}$}

\section{Une loi du 24 mai 1951}

La loi du 24 mai 1951 modifie l'ordonnance du 2 février 1945 sur l'enfance délinquante et abroge la vieille loi du 5 août 1850 qui admettait le placement des mineurs condamnés dans les colonies pénitentiaires et correctionnelles devenues maisons d'éducation surveillée en 1927, puis institutions publiques d'éducation surveillée en 1940.

Cette loi de 1951 prévoit toutefois dans son article 23 que ces mineurs pourront être placés dans des établissements spéciaux pour des peines supérieures à dix mois. Un décret du 12 avril 1952 donnera un statut à ces établissements spéciaux : institutions spéciales d'éducation surveillée (ISES). Les jeunes condamnés devront avoir moins de 20 ans. Si leur peine est inférieure à 12 mois, ils n'iront pas en ISES, ils seront regroupés dans des quartiers de mineurs de maison d'arrêt. Nous nous trouvons là, pour les peines les plus longues, dans une logique de peine éducative, celle développée au cours du XIXème siècle et consacrée par la loi du 5 août 1850 .

La direction de l'Éducation surveillée semble très réticente à cette réforme qui entretient la confusion entre peine et mesure éducative, elle ouvre deux ISES, celle de Lesparre pour les filles le 1er août 1952, celle des Sables d'Olonne pour les garçons en octobre 1954, dans deux prisons désaffectées prêtées par l'Administration pénitentiaire, mais elle n'y recevra aucun mineur condamné. On ne trouvera à Lesparre, comme aux Sables d'Olonne, que « des mineurs qui se sont montrés absolument réfractaires à une rééducation à long terme en internat ", ${ }^{(1)}$ ou des mineurs sortant de prison après à un séjour en détention préventive. En fait, l'ISES permettra à la direction de l'Éducation surveillée de trouver pour les mineurs les plus difficiles - on parle alors d'inéducables - un mode de réponse éducative autre que celui de l'internat de $\left.{ }^{*}\right)$ Article publié

dans le Temps de l'histoire, $\mathrm{n}^{\circ} 1$, février 1998

(1) Rapport annuel, direction de l'Éducation surveillée, 1952. 
(2) Paul Lutz,

" Les systèmes pédagogiques dans l'internat de rééducation ", Revue pénitentiaire et de droit pénal, $\mathrm{n}^{\circ} 7-9$, juilletseptembre 1957, p. 591.

(3) Ibid., p. 597. rééducation, type IPES, reposant sur un placement de trois ans lié à la durée de la formation professionnelle, mais aussi à des nécessités d'éloignement et d'exclusion souhaités par l'environnement social.

C'est vraisemblablement pour des raisons budgétaires que la direction de l'Éducation surveillée accepte de mener ces deux expériences dans des prisons désaffectées : les moyens financiers de l'Éducation surveillée seront très modiques jusqu'en 1962, date de son inscription au IVème Plan. On ne peut toutefois exclure l'idée que, pour des mineurs réputés difficiles, le choix de deux anciennes prisons ait un lien avec des préoccupations de sécurité.

\section{Le projet de Paul Lutz et l'expérience de Lesparre}

Avant de devenir en 1952 la première ISES, l'institution de Lesparre, ouverte dans une prison désaffectée, était, depuis 1949, l'annexe de l'ancienne école de préservation de Cadillac, un établissement pour filles au lourd passé pénitentiaire qui ne fermera ses portes qu'en octobre 1951. Les dernières adolescentes de Cadillac se retrouveront, fin 1951-début 1952, à Lesparre.

Paul Lutz, magistrat et inspecteur à la direction de l'Éducation surveillée dès 1945, s’intéresse aux méthodes pédagogiques à mettre en place pour les jeunes de l'Éducation surveillée et plus particulièrement pour les filles. Il s'efforcera d'aider à la réforme de Cadillac qui s'avère impossible; il contribue à la création de l'IPES de Brécourt. C'est lui qui élabore, à partir de Lesparre en 1952, le modèle qui va préfigurer l'ISES.

Ce que souhaite Paul Lutz, qui a connu l'Éducation surveillée à l'intérieur de l'Administration pénitentiaire, c'est « introduire dans la réalité la distinction et l'opposition formulées par la loi entre mesure éducative et peine $» .{ }^{(2)}$ Dans les années 1950, Paul Lutz est particulièrement intéressé par les réponses éducatives que l'on pourrait faire intervenir valablement auprès des jeunes considérés comme inéducables par les institutions de rééducation.

Influencé par la psychopédagogie, la psychanalyse et les travaux de l'autrichien Aïchorn auprès des mineurs délinquants, il recherche des méthodes rééducatives qui ne se servent plus de l'intimidation ou de l'action des groupes, mais surtout de la psychologie des profondeurs : "Chaque être doit être traité individuellement selon ses besoins et ses possibilités propres sans référence à des normes générales ou à une collectivité. " ${ }^{(3)}$ 
L'autre idée soutenue par Paul Lutz est que l'enfant qui a de graves difficultés de comportement n'a pas nécessairement besoin d'une discipline accrue; au contraire, il faut lui proposer des exigences à sa mesure et le conduire progressivement vers la normalité en lui évitant au maximum les situations d'échec.

C'est à partir de cette approche que Paul Lutz a l'idée d'un établissement à très petit effectif dans lequel " la sauvegarde de la collectivité ne devient jamais le motif des exigences opposées au jeune ". ${ }^{(4)}$ C'est ce qui l'amènera à fermer Cadillac, trop imprégnée de principes pénitentiaires, et à élaborer le projet de Lesparre en y privilégiant un traitement de type individuel pour des adolescentes de 17 ans.

L'ISES de Lesparre est prévue pour 17 jeunes; le séjour y est d'environ 6 mois; la mineure y reçoit tout d'abord une formation ménagère de quelques semaines, après quoi on lui cherche un apprentissage artisanal dans les environs. À l'issue des 6 mois, l'adolescente fait l'objet d'un service de suite qui s'occupe de sa réinsertion sociale sous le contrôle du juge des enfants de Bordeaux et en collaboration avec le service de psychiatrie infantile de Bordeaux.

Chaque mineure, explique Paul Lutz, doit être traitée suivant sa personnalité, d'où la nécessité de recruter des éducateurs, des psychologues, des médecins à même d'utiliser " toutes les ressources de la pédagogie et des autres techniques pour traiter les déficiences biologiques et psychiques et les comportements souvent inhabituels ". ${ }^{(5)} \mathrm{Il}$ est toutefois précisé que l'établissement ne reçoit pas de " cas typiquement psychiatriques ». C'est la première fois qu'à la direction de l'Éducation surveillée, on évoque la pluridisciplinarité des équipes ailleurs que dans le domaine de l'observation.

Dans son rapport de 1956, la direction de l'Éducation surveillée confirme que, pour les mineures de 17 ans qui se présentent à la rééducation avec un pronostic comportant " des difficultés exceptionnelles ", une pédagogie spéciale qui aboutit à l'abandon de la référence à la vie de groupe et à l'aménagement d'établissements à effectifs réduits est à développer.

Il s'agit de créer " des petites collectivités dépourvues de réglementation générale où les exigences se feront surtout en fonction des individus et des moments divers de leur vie ». "Plus un mineur est gravement atteint, moins il supporte un système disciplinaire " ${ }^{\left({ }^{(}\right)}$souligne Paul Lutz.
(4) Ibid.

(5) Paul Lutz, Rapport d'inspection sur l'ISES des Sables d'Olonne, 1956, document consulté à la bibliothèque $2 \mathrm{RJ}$, CNFE-PJJ, Vaucresson.

(6) Ibid. 
On est là dans une logique qui est beaucoup plus celle de la cure, du traitement. On conçoit que, dans cette optique, l'Éducation surveillée ne recevra pas de mineurs condamnés. Avec l'ISES, la rééducation entre dans un processus d'individualisation de la prise en charge, une démarche qui préfigure avec vingt ans d'avance les orientations de l'Éducation surveillée de 1975, lorsqu'elle reparlera d'ISES.

L'ambivalence à l'égard de ces jeunes « difficiles » subsiste. Comme à Lesparre, c'est dans une prison désaffectée que l'on tentera aux Sables d'Olonne, en 1954, l'expérience de l'ISES pour les garçons.

\section{L'ISES des Sables d'Olonne}

L'histoire très courte de cette institution (1954-1960) fait l'objet de plusieurs rapports d'inspection de la direction de l'Éducation surveillée qui témoignent de l'intérêt que la direction portait à cette expérience. Les premières inspections ont été faites par Paul Lutz.

L'établissement ouvre en octobre 1954. On sait peu de choses sur les premiers mois de son existence. Le directeur vient du centre d'observation de Lyon; c'est un ancien instituteur; deux éducateurs venant d'IPES lui sont adjoints. L'institution reste très carcérale. Ce sont les trois premiers élèves arrivés en octobre et novembre qui collaborent à la rénovation des locaux et à leur entretien. Un atelier de fabrication de parpaings est rapidement ouvert, ce qui permet de donner un salaire aux élèves qui suivent des cours par correspondance avec la FPA. En janvier 1955, lors de la première inspection de Paul Lutz, trois nouveaux élèves sont attendus; le projet prévoit pour eux des sorties en ville, au cinéma avec un éducateur. Ils ont même été invités à une soirée par le maire adjoint, ce qui laisse augurer des bonnes intentions de la municipalité et du souci d'ouverture sur l'environnement de la part du directeur de l'établissement.

Pour des raisons de santé, ce premier directeur est remplacé en juillet 1955 par Pierre Degoul qui vient de l'IPES de Saint-Maurice où il est chef de service éducatif; il a été contacté par Paul Lutz.

À l'arrivée de P. Degoul, l'effectif du personnel a légèrement augmenté : 3 éducateurs venant d'IPES, un agent auxiliaire, une cuisinière. Lors de la seconde inspection de Paul Lutz, le 18 juillet 1955, 10 mineurs sont à l'établis- 
sement et 5 en postcure chez des artisans locaux, dont un embarqué sur un thonier. Le séjour à l'établissement est d'environ 6 mois. Les jeunes en postcure prennent en charge, grâce au salaire gagné pendant leur séjour, leur vêture; trois d'entre eux paient une chambre en ville, par contre ils continuent à prendre leurs repas à l'institution. C'est à partir de l'arrivée de Pierre Degoul que s'ouvrent réellement les portes de l'ancienne prison, que l'on supprime les barreaux aux fenêtres. Comme les chambres de postcure sont difficiles à trouver aux Sables d'Olonne, le directeur envisage de créer 4 chambres indépendantes à l'intérieur de l'ISES avec une porte d'accès à l'extérieur. On parlera de " home ». La majorité des jeunes sont inscrits dans des sociétés locales d'athlétisme. Le dimanche, les élèves sortent en groupe, mais il peut y avoir des sorties libres entre 15 et 20 heures pour les plus méritants.

En août 1956, Paul Lutz fait une nouvelle inspection, il y a maintenant 6 membres du personnel, dont un instructeur technique qui ouvre un atelier de menuiserie et une éducatrice venant du service de liberté surveillée qui a la charge de la postcure. 14 mineurs sont à l'ISES, 4 sont en postcure au foyer de Nantes qui vient d'ouvrir et 11 en postcure chez des employeurs des Sables d'Olonne ou de la région, certains logent au " home ».

Dans une conférence de septembre 1957 à des personnalités locales, Pierre Degoul explique la nature du projet et le type de jeunes reçus dans l'établissement : "Quelques débiles, des caractériels, des psychopathes..., nous avons des garçons indésirables après de multiples incidents, ils ont fait l'objet de nombreux transferts d'établissement à établissement, avec quelques séjours en maison d'arrêt ou en asile psychiatrique. " "(7) Il évoque aussi les "faux psychopathes » : " Ils ont acquis leurs troubles au cours d'une longue carrière dans les orphelinats ou les internats de rééducation. " ${ }^{(8)} \mathrm{Il}$ ne s'agit pas, précise-t-il, de "troubles constitutionnels ${ }^{(9)}$ et il cite quelques mineurs placés à l'ISES dont la moyenne de placements dépasse la dizaine depuis la petite enfance.

Le but de l'ISES, explique Pierre Degoul, « c'est de créer un climat où leur personnalité pourra se manifester sans risque de provoquer des incidents tout en essayant de les resocialiser $»{ }^{(10)}$

C'est alors qu'il insiste sur l'importance de la " postcure ", ce temps qui suit le passage après quelques mois en institution. Questionné sur la nature de la cure, il répond que « ce qui a été tenté dans les nombreux établissements où
(7) Conférence de Pierre Degoul, les Sables d'Olonne, septembre 1957, document consulté à la bibliothèque $2 \mathrm{RJ}$, CNFE-PJJ, Vaucresson.
(8) Ibid.
(9) Ibid.
(10) Ibid. 
(11) Ibid.

(12) Ibid.

(13) Ibid

(14) Ibid.

(15) Ibid

le mineur est passé s'est manifesté le plus souvent par un échec; les jeunes se révélaient le plus souvent inaptes au régime collectif et à une pédagogie centrée sur le groupe, telle qu'elle se développe dans les IPES .(11) $^{(1)}$

Lorsque le jeune arrive aux Sables d'Olonne, il a souvent 18 ans. Il ne s'agit pas, précise Pierre Degoul, «de reprendre une cure, le séjour à l'institution doit être considéré comme un complément d'observation ", en donnant à ce terme le sens « d'exploitation d'expériences antérieures qui n'ont pas toutes été sans influence bénéfique ». ${ }^{(12)}$

Le projet de l'ISES est en fait de chercher une solution qui paraisse adaptée à la situation du garçon, « une période préparatoire à la postcure avec des essais de resocialisation ". ${ }^{(13)}$ Le directeur de l'ISES insiste beaucoup sur cette préparation matérielle à la postcure : « Lui permettre d'acquérir un trousseau, des outils de travail, il faut qu'il puisse couvrir progressivement ses frais de logement, de nourriture. ${ }^{(14)}$

Une grande importance est accordée aux conditions nécessaires à une indépendance matérielle, "conditions mêmes de l'accession au statut d'adulte ". ${ }^{(15)}$ C'est une des raisons pour lesquelles Pierre Degoul n'est pas favorable, dans la postcure, au placement dans des familles d'accueil. Il juge cette solution inadaptée à des mineurs qui ont 18 ans et plus.

Si la notion d'autonomie matérielle est largement développée dans le projet, on n'évoque pas la capacité d'autonomie personnelle du jeune à assumer une situation d'indépendance; c'est vraisemblablement là une des failles du projet.

Pierre Degoul distingue dans son projet deux grandes catégories d'élèves :

- ceux qui pourront être reçus dans leur famille en permission renouvelable après une année de séjour à l'ISES et en postcure.

Après un séjour d'environ 6 mois à l'établissement, débute la période de postcure qui se fera souvent par étapes; tout d'abord un placement professionnel à l'extérieur avec obligation de réintégrer l'établissement où le jeune est logé et nourri. Si l'expérience se révèle satisfaisante, le jeune pourra être logé au "home" attenant à l'institution, ensuite il pourra disposer d'une chambre en ville, avec des permissions renouvelables chez lui au bout d'un an. On reste dans une optique pédagogique qui s'apparente au système progressif tel qu'il est encore très en vigueur dans les IPES. L’amélioration du comportement du jeune, sa faculté de s'adapter à la norme sociale conditionnent l'évolution de 
son mode de vie. À la différence de l'IPES où cette évolution est très tributaire des règles institutionnelles, aux Sables d'Olonne, le jeune est très vite confronté à la vie sociale à l'extérieur.

- ceux dont les familles ne présentent pas des garanties suffisantes, ou ceux qui ne désirent pas retourner dans leur famille. N'oublions pas que nombre de jeunes qui sortent de l'ISES ont 19 ans et plus.

Le retour dans la famille n'étant pas envisagé, il est prévu la création d'un foyer de semi-liberté. Il ouvrira à Nantes, rue Félix Thomas, en juillet 1956. Ce sera le premier foyer créé par la direction de l'Éducation surveillée. Sous l'autorité du directeur de l'ISES, la responsabilité de ce foyer est confiée à un couple d'éducateurs, M. et Mme Foucher, qui ont travaillé antérieurement à l'internat approprié de Chanteloup, établissement d'éducation surveillée réservé aux mineurs de moins de 13 ans. M. et Mme Foucher sont inscrits au foyer de Nantes dans un rôle de substitut parental, Pierre Degoul évoque la fonction de "substitut paternel " de l'éducateur et celle de " maman " de l'éducatrice. Il insiste sur ces références qui lui semblent tout à fait convenir pour des jeunes le plus souvent "abandonniques». M. et Mme Foucher, qui vivent au foyer avec leurs trois petites filles, présentent le modèle de la famille idéale; il semble que ce fut, pour bien des jeunes venus des Sables d'Olonne et qui passèrent souvent un an et plus au foyer, un lieu de réassurance et de restructuration. C'est en tout cas le souvenir qui est gardé de cette expérience par les personnels qui en ont connu les débuts.

Une inspection d'Henri Gaillac, en novembre 1957, correspond à l'heure des premiers bilans. Les rapports avec la municipalité se sont tendus; 6 infractions ont été imputables aux mineurs de l'institution en 1956 et 7 depuis le début 1957; le plus souvent, elles sont commises par des jeunes qui viennent d'arriver et dont certains ne resteront pas.

Depuis l'ouverture en 1954, 76 mineurs ont été affectés à l'ISES, pour la moitié d'entre eux, suite à des "vols graves, à des cambriolages avec effraction ». ${ }^{(16)}$ Parmi les 50 mineurs qui ont quitté l'ISES depuis l'ouverture, on distingue deux types d'évolution :

- 19 sont sortis après moins de 3 mois et le plus souvent après quelques jours (fugue, récidive...); parmi eux, 17 sont en maison d'arrêt, ou en hôpital psychiatrique.
(16) Henri Gaillac,

Rapport d'inspection sur les Sables d'Olonne, novembre 1957 , document consulté à la bibliothèque 2RJ, CNFEPJJ, Vaucresson. 
(17) Entretien de Jacques Bourquin avec M. Girault, 1997.

(18) Henri Gaillac, Rapport d'inspection..., op. cit.

(19) Ibid.

(20) Ibid
- les 31 autres, qui ont fait un séjour d'au moins six mois à l'institution, suivi d'une postcure, sont, pour 20 d'entre eux, " resocialisés ", et pour 10, " en voie d'amélioration "; un seul se trouve en maison d'arrêt. Il semblerait, d'après ce constat du rapport d'inspection, que ceux qui ont accepté le projet de l'institution évoluent favorablement au moins dans l'année ou les deux années qui suivent le passage à l'ISES.

Les 26 mineurs qui relèvent de l'institution en novembre 1957 sont, pour 13 d'entre eux, présents à l'établissement; les 13 autres sont en postcure au foyer de Nantes ou aux Sables d'Olonne.

Le projet n'a pas subi de modifications particulières depuis l'origine. Les élèves continuent à bénéficier de sorties libres aux Sables d'Olonne, et ceci dès un mois après leur arrivée. Les quelques délits commis en 1956 et 1957 ont toutefois altéré les relations avec la municipalité. Le député-maire évoque la nécessité de la fermeture de l'établissement; il est suivi en cela par le président du tribunal civil des Sables d'Olonne. Ce n'est pas l'avis du juge des enfants de la Roche-sur-Yon, M. Girault, qui dit « apprécier beaucoup l'action du directeur de l'ISES ». ${ }^{(17)}$ Henri Gaillac conclut dans son rapport que la méthode de rééducation des Sables d'Olonne n'est pas à remettre en cause : "Elle remplit bien sa fonction de réinsertion. " ${ }^{(18)}$ Il ne lui semble pas que la situation de l'établissement dans une station balnéaire « aggrave sérieusement le risque éducatif " ${ }^{(19)}$; il cite à ce sujet l'avis du sous-préfet des Sables d'Olonne qui dit " que la station balnéaire est plus néfaste aux garçons de l'Éducation surveillée, qu'ils ne sont nuisibles à celle-ci ". ${ }^{(20)}$

En mai 1958, l'ISES a reçu 80 élèves, soit seulement 4 depuis novembre 1957 ; on assiste manifestement à un ralentissement des admissions. La direction de l'Éducation surveillée envisage la nomination d'un chef de service éducatif aux Sables d'Olonne, Pierre Degoul prenant à Nantes la direction de l'ensemble foyer-consultation-ISES. C'est l'amorce des consultations d'orientation et d'action éducative (COAE).

\section{Vers la fermeture}

Au cours de la période estivale de 1959, les rapports avec la population vont se dégrader. Le 28 juillet, 3 mineurs de l'ISES agressent 2 petites filles; une infraction d'attentat à la pudeur est relevée. A cela s'ajoute une bagarre 
avec de jeunes estivants. Il est à remarquer que, pendant cette période de vacances, l'atelier de menuiserie de l'ISES est fermé; les élèves sont plutôt désœuvrés; il n’y a que deux éducateurs.

Le sous-préfet organise une réunion des autorités municipales. Un article paraît sous un gros titre dans le France Soir du 30 juillet 1959 : "Aux Sables d'Olonne, des jeunes délinquants en promenade surveillée mettent $\mathrm{KO}$ cinq estivants avec des gourdins, des tessons de bouteille et des couteaux. " La direction de l'Éducation surveillée envoie un inspecteur, M. Joseph, qui propose que toute affectation de jeunes soit suspendue jusqu'au 15 septembre et que, par la suite, " la sélection des jeunes affectés à l'ISES soit plus poussée ». (21)

Le 2 octobre 1959, suite à une demande de la direction de l'Éducation surveillée, le chef de service éducatif de l'ISES, apparemment sans en informer son directeur Pierre Degoul, envoie un projet de réorganisation de l'institution auquel il donne le nom de "maison disciplinaire »; le placement à l'ISES, conclut-il, constitue une sanction.

«Les portes seront fermées, les garçons n'auront aucun contact avec l'extérieur... la durée du séjour ne devra pas durer plus de six mois ».(22)

L'effectif des mineurs passera de 14 à 24 . On augmentera d'une unité les deux ateliers existants.

Ce nouveau projet apparaît en totale contradiction avec celui de 1954. Le rédacteur insiste sur le fait que "les mineurs confiés à l'institution ne sortiront que de manière exceptionnelle ».(23)

On aboutit à une expérimentation de centre fermé qui semble, pour les mineurs " difficiles " de l'Éducation surveillée, s'inscrire entre l'IPES de type classique et le recours à la prison.

Ce projet ne sera pas vraiment réalisé et l'ISES fermera définitivement au cours du premier semestre 1960 .

Dans le même temps, l'ISES de Lesparre disparaît, mais les raisons en sont différentes. Depuis son ouverture, les résultats auprès de 200 adolescentes qui y ont séjourné ont été positifs pour la majorité d'entre elles. La direction de l'Éducation surveillée souhaite poursuivre l'expérience, mais dans un autre cadre que celui d'une prison désaffectée. Une villa est achetée à Corenc, près de Grenoble, mais 4 ans se dérouleront entre la fermeture de Lesparre et l'ouverture de Corenc; entre temps le projet se sera modifié; on ne parlera plus d'ISES.
(21) M. Joseph,

Rapport d'inspection sur les Sables d'Olonne, septembre 1959, document consulté à la bibliothèque 2RJ, CNFEPJJ, Vaucresson.

(22) Rapport de R. Labasque, chef de service éducatif de l'ISES des Sables d'Olonne, au directeur de l'Éducation surveillée, 2 octobre 1959, document consulté à la bibliothèque 2RJ, CNFEPJJ, Vaucresson.

(23) Ibid. 
(24) Paul Lutz « Les systèmes édagogiques..." ", op. cit., p. 598.
Le projet de centre fermé auquel aboutit l'ISES des Sables d'Olonne ne se réalisera en fait, sur des bases bien différentes, que dix ans plus tard avec la création par le Dr Roumajon du centre fermé de Vauhallan. Cette institution, qui relève du secteur associatif et à laquelle collabore l'Éducation surveillée, reçoit une trentaine de mineures «borderline " gravement inadaptées et souvent multirécidivistes. Le projet donne une grande importance à la dimension thérapeutique; l'équipe est à la fois médicale, psychologique et éducative. Face aux difficultés rencontrées aussi bien financières que pédagogiques, il est mis fin à ce type d'intervention en 1974.

Parallèlement à cette expérience, la direction de l'Éducation surveillée fera fonctionner, entre 1970 et 1979, deux centres d'observation de sécurité à Juvisysur-Orge et à Épernay. Ce sont des structures fermées pour adolescents le plus souvent récidivistes. L'échec de ces institutions progressivement plus sécuritaires qu'éducatives amènera à la modification du projet et à l'abandon par la direction de l'Éducation surveillée du modèle d'établissement fermé.

\section{Pour conclure}

L'abandon du projet des Sables d'Olonne est indéniablement lié aux incidents de 1959, en une période où le phénomène des bandes, les « blousons noirs ", et l'augmentation de la délinquance juvénile sont largement commentés par la presse et préoccupent de plus en plus l'opinion publique.

Alors que le général de Gaulle a créé en 1958, dans un objectif de prévention, un ministère de la Jeunesse, la direction de l'Éducation surveillée réinvestit pour certains adolescents récidivistes les quartiers de mineurs dans les prisons (Fresnes, Lyon, Marseille) et s'inscrit dans une logique sécuritaire. Une autre raison du relatif échec des Sables d'Olonne et de sa fermeture est liée à une lacune importante du projet, l'inexistence de sa référence psychologique. Paul Lutz avait souligné l'importance qu'il y avait pour ces mineurs difficiles, « qui ne sont pas de simples sous-éduqués ", ${ }^{(24)}$ de mener une action individualisée dans ses dimensions psychologiques et éducatives. Il évoquait la nécessité d'un recrutement adapté et d'une formation poussée des personnels de l'ISES. Ce ne fut pas le cas. La pratique des Sables d'Olonne n'a eu qu'une dimension éducative menée par des éducateurs, avec pour seule expérience celle des gros internats de rééducation dont les projets étaient bien différents de celui de l'ISES. 
En 1955, l'idée de pluridisciplinarité des équipes n’est présente à l'Éducation surveillée que dans les centres d'observation. Une psychologue sera nommée à Nantes en 1957, mais ce sera dans le cadre de la nouvelle consultation d'orientation éducative et elle n'interviendra quasiment pas aux Sables d'Olonne. Néanmoins, l'ISES des Sables d'Olonne, comme celle de Lesparre, furent des initiatives qui préfigurent, avec près de vingt ans d'avance, les ISES que l'Éducation surveillée créera à partir de 1973. On insistera beaucoup dans ces premières ISES sur la nécessité d'adapter leur réponse aux besoins du jeune, en prenant le contre-pied du projet de l'IPES qui, dans une logique de normalisation, conditionnait le jeune à s'adapter à l'institution. Enfin, et c'est là une des innovations des Sables d'Olonne et de Lesparre, la réponse apportée par l'institution à ces jeunes aux comportements difficiles ne devait pas aller dans le sens d'une discipline accrue mais, pour reprendre la formulation de Paul Lutz, il s'agissait de " leur proposer des exigences à leur mesure en leur évitant les situations d'échec ». ${ }^{(25)}$ 